\title{
Optimal Control Strategy for SEIR with Latent Period and a Saturated Incidence Rate
}

\author{
Abta Abdelhadi ${ }^{1}$ and Laarabi Hassan ${ }^{2}$ \\ ${ }^{1}$ Department of Mathematics and Computer Science, Faculty of Sciences El Jadida, Chouaib Doukkali University, \\ P.O. Box 20, El Jadida, Morocco \\ ${ }^{2}$ Department of Mathematics and Computer Science, Faculty of Sciences Ben M'sik, Hassan II University, \\ P.O. Box 7955, Sidi Othman, Casablanca, Morocco
}

Correspondence should be addressed to Abta Abdelhadi; abtaabdelhadi@yahoo.fr and Laarabi Hassan; hlaarabi@yahoo.fr

Received 24 April 2013; Accepted 22 May 2013

Academic Editors: Q. Song and Y. Wang

Copyright (C) 2013 A. Abdelhadi and L. Hassan. This is an open access article distributed under the Creative Commons Attribution License, which permits unrestricted use, distribution, and reproduction in any medium, provided the original work is properly cited.

\begin{abstract}
We propose an SEIR epidemic model with latent period and a modified saturated incidence rate. This work investigates the fundamental role of the vaccination strategies to reduce the number of susceptible, exposed, and infected individuals and increase the number of recovered individuals. The existence of the optimal control of the nonlinear model is also proved. The optimality system is derived and then solved numerically using a competitive Gauss-Seidel-like implicit difference method.
\end{abstract}

\section{Introduction}

Epidemiological models with latent or incubation period have been studied by many authors because many diseases have a latent or incubation period, during which the individual is said to be infected but not infectious. This period can be modeled by introducing an exposed class [1] Therefore, it is an important subject to determine the optimal vaccination strategies for the models which take into account the incubation period.

In this paper, our aim is to set up an optimal control problem related to the SEIR epidemic model. The dynamics of this model are governed by the following equations $[2,3]$ :

$$
\begin{gathered}
\frac{d S}{d t}=A-\mu S(t)-\frac{\beta S(t) I(t)}{1+\alpha_{1} S(t)+\alpha_{2} I(t)}, \\
\frac{d E}{d t}=\frac{\beta S(t) I(t)}{1+\alpha_{1} S(t)+\alpha_{2} I(t)}-(\sigma+\mu) E(t), \\
\frac{d I}{d t}=\sigma E(t)-(\mu+\alpha+\gamma) I(t), \\
\frac{d R}{d t}=\gamma I(t)-\mu R(t),
\end{gathered}
$$

where $S$ is the number of the susceptible individuals, $E$ is the number of exposed individuals, $I$ is the number of infected individuals, $R$ is the number of the recovered individuals, $A$ is the recruitment rate of the population, $\mu$ is the natural death of the population, $\alpha$ is the death rate due to disease, $\beta$ is the transmission rate, $\alpha_{1}$ and $\alpha_{2}$ are the parameter that measure the inhibitory effect, $\gamma$ is the recovery rate of the infective individuals, and $\sigma$ is the rate at which exposed individuals become infectious. Thus $1 / \sigma$ is the mean latent period.

Now we introduce one control $u(t)$ which represents the percentage of susceptible individuals being vaccinated per unit of time. Hence, (1) becomes

$$
\begin{gathered}
\frac{d S}{d t}=A-\mu S(t)-\frac{\beta S(t) I(t)}{1+\alpha_{1} S(t)+\alpha_{2} I(t)}-u(t) S(t), \\
\frac{d E}{d t}=\frac{\beta S(t) I(t)}{1+\alpha_{1} S(t)+\alpha_{2} I(t)}-(\sigma+\mu) E(t), \\
\frac{d I}{d t}=\sigma E(t)-(\mu+\alpha+\gamma) I(t), \\
\frac{d R}{d t}=\gamma I(t)-\mu R(t)+u(t) S(t) .
\end{gathered}
$$


In addition, for biological reasons, we assume that the initial data for system (2) satisfy

$$
\begin{array}{ll}
S(0)=S_{0} \geq 0, & E(0)=E_{0} \geq 0, \\
I(0)=I_{0} \geq 0, & R(0)=R_{0} \geq 0 .
\end{array}
$$

The rest of the paper is organized as follows. In Section 2, we use Pontryagin's maximum principle to investigate analysis of control strategies and to determine the necessary conditions for the optimal control of the disease. Mathematical results are illustrated by numerical simulations in Section 3. Finally, we summarize our work and propose the future focuses.

\section{The Optimal Control Problem}

The optimal control problem is to minimize the objective (cost) functional given by

$$
J(u)=\int_{0}^{t_{\text {end }}}\left[A_{1} S(t)+A_{2} E(t)+A_{3} I(t)+\frac{1}{2} \tau u^{2}(t)\right] d t
$$

subject to the differential equations (2), where the first tree terms in the functional objective represent benefit of $S(t)$, $E(t)$, and $I(t)$ populations that we wish to reduce, and the parameters $A_{1}, A_{2}$, and $A_{3}$ are positive constants to keep a balance in the size of $S(t), E(t)$, and $I(t)$, respectively. We use in the second term in the functional objective (as it is customary) the quadratic term $(1 / 2) \tau u^{2}$, where $\tau$ is a positive weight parameter which is associated with the control $u(t)$, and the square of the control variable reflects the severity of the side effects of the vaccination.

Our target is to minimize the objective functional defined in (4) by decreasing the number of infected, exposed, and susceptible individuals and increasing the number of recovered individuals by using possible minimal control variables $u(t)$. In other words, the control variable $u(t) \in U_{\text {ad }}$ represents the percentage of susceptible individuals being vaccinated per unit of time and $U_{\text {ad }}$ is the control set defined by

$U_{\text {ad }}=\left\{u \mid u(t)\right.$ is measurable, $\left.0 \leq u(t) \leq u_{\max }<\infty, t \in\left[0, t_{\text {end }}\right]\right\}$.

2.1. Existence of an Optimal Control. For the existence of an optimal control we use the result in Lukes [4], and we obtain the following theorem.

Theorem 1. There exists a control function $u^{*}(t)$ so that

$$
J\left(u^{*}(t)\right)=\min _{u \in U} J(u(t)) .
$$

Proof. To prove the existence of an optimal control it is easy to verify that

(1) the set of controls and corresponding state variables is nonempty,

(2) the admissible set $U_{\text {ad }}$ is convex and closed,

(3) the right hand side of the state system (2) is bounded by a linear function in the state and control variables,
(4) the integrand of the objective functional is convex on $U_{\text {ad }}$,

(5) there exist constants $\omega_{1}>0$ and $\omega_{2}>0$, and $\rho>$ 1 such that the integrand $L(S, E, I, u)$ of the objective functional satisfies $\rho>1$ and positive numbers $\omega_{1}$ and $\omega_{2}$ such that $L(S, E, I, u) \geq \omega_{2}+\omega_{1}\left(|u|^{2}\right)^{\rho / 2}$.

The result follows directly from [5].

2.2. Characterization of the Optimal Control. Before characterizing the optimal control, we first define the Lagrangian for the optimal control problem (2) and (4) by

$$
L(S, E, I, u)=A_{1} S(t)+A_{2} E(t)+A_{3} I(t)+\frac{1}{2} \tau u^{2}(t)
$$

and the Hamiltonian $H$ for the control problem by

$$
\begin{aligned}
& H\left(S, E, I, R, u, \lambda_{1}, \lambda_{2}, \lambda_{3}, \lambda_{4}, t\right) \\
&= L(S, E, I, u)+\lambda_{1} \frac{d S(t)}{d t} \\
&+\lambda_{2} \frac{d E(t)}{d t}+\lambda_{3} \frac{d I(t)}{d t}+\lambda_{4} \frac{d R(t)}{d t},
\end{aligned}
$$

where $\lambda_{1}, \lambda_{2}, \lambda_{3}$, and $\lambda_{4}$ are the adjoint functions to be determined suitably. Next, by applying Pontryagin's maximum principle [6] to the Hamiltonian, we obtain the following theorem.

Theorem 2. Given an optimal control $u^{*}(t)$ and solutions $S^{*}(t), E^{*}(t), I^{*}(t)$, and $R^{*}(t)$ of the corresponding state system (2) and (4), there exists adjoint variables $\lambda_{1}, \lambda_{2}, \lambda_{3}$, and $\lambda_{4}$ that satisfy

$$
\begin{aligned}
\frac{d \lambda_{1}(t)}{d t}= & -A_{1}+\lambda_{1}(t)\left(\mu+u^{*}(t)+\Lambda_{1}\right) \\
& -\lambda_{2}(t) \Lambda_{1}-\lambda_{3}(t) u^{*}(t), \\
\frac{d \lambda_{2}(t)}{d t}= & -A_{2}+\lambda_{2}(t)(\mu+\sigma)-\lambda_{3}(t) \sigma, \\
\frac{d \lambda_{3}(t)}{d t}= & -A_{3}+\lambda_{1}(t) \Lambda_{2}-\lambda_{2}(t) \Lambda_{2} \\
& +\lambda_{3}(t)(\mu+\alpha+\gamma)-\lambda_{4}(t) \gamma, \\
& \frac{d \lambda_{4}(t)}{d t}=\lambda_{3}(t) \mu,
\end{aligned}
$$

where $\Lambda_{1}=\left(\beta I^{*}\left(1+\alpha_{2} I^{*}\right) /\left(1+\alpha_{1} S^{*}+\alpha_{2} I^{*}\right)^{2}\right)$ and $\Lambda_{2}=\left(\beta S^{*}\left(1+\alpha_{1} S^{*}\right) /\left(1+\alpha_{1} S^{*}+\alpha_{2} I^{*}\right)^{2}\right)$ with transversality conditions

$$
\lambda_{i}\left(t_{\text {end }}\right)=0, \quad i=1,2,3,4 .
$$

Furthermore, the optimal control $u^{*}(t)$ is given by

$$
u^{*}(t)=\max \left(\min \left(\frac{\left(\lambda_{1}(t)-\lambda_{4}(t)\right) S^{*}(t)}{\tau}, u_{\max }\right), 0\right) .
$$


Proof. Using the Pontryagin's maximum principle we obtain the adjoint equations and transversality conditions such that

$$
\begin{gathered}
\frac{d \lambda_{1}(t)}{d t}=-\frac{\partial H}{\partial S} \\
=-A_{1}+\lambda_{1}(t)\left(\mu+u^{*}(t)+\Lambda_{1}\right) \\
-\lambda_{2}(t) \Lambda_{1}-\lambda_{3}(t) u^{*}(t), \\
\frac{d \lambda_{2}(t)}{d t}=-\frac{\partial H}{\partial E}=-A_{2}+\lambda_{2}(t)(\mu+\sigma)-\lambda_{3}(t) \sigma \\
\frac{d \lambda_{3}(t)}{d t}=-\frac{\partial H}{\partial I} \\
=-A_{3}+\lambda_{1}(t) \Lambda_{2}-\lambda_{2}(t) \Lambda_{2} \\
\quad+\lambda_{3}(t)(\mu+\alpha+\gamma)-\lambda_{4}(t) \gamma, \\
\frac{d \lambda_{4}(t)}{d t}=-\frac{\partial H}{\partial R}=\lambda_{4}(t) \mu,
\end{gathered}
$$

and by using the optimality conditions we find

$$
\begin{array}{r}
\frac{\partial H}{\partial u}=\tau u^{*}(t)-\lambda_{1}(t) S^{*}+\lambda_{4}(t) S^{*}=0, \\
\text { at } u=u^{*}(t) \text { which gives } \\
u^{*}(t)=\frac{\left(\lambda_{1}(t)-\lambda_{4}(t)\right) S^{*}(t)}{\tau} .
\end{array}
$$

Using the property of the control space, we obtain

$$
\begin{gathered}
u^{*}(t)=0 \quad \text { if } \frac{\left(\lambda_{1}(t)-\lambda_{4}(t)\right) S^{*}(t)}{\tau} \leq 0, \\
u^{*}(t)=\frac{\left(\lambda_{1}(t)-\lambda_{4}(t)\right) S^{*}(t)}{\tau} \\
\text { if } 0<\frac{\left(\lambda_{1}(t)-\lambda_{4}(t)\right) S^{*}(t)}{\tau}<u_{\max }, \\
u^{*}(t)=u_{\max } \text { if } \frac{\left(\lambda_{1}(t)-\lambda_{4}(t)\right) S^{*}(t)}{\tau} \geq u_{\max } .
\end{gathered}
$$

So the optimal control is characterized as

$$
u^{*}(t)=\max \left(\min \left(\frac{\left(\lambda_{1}(t)-\lambda_{4}(t)\right) S^{*}(t)}{\tau}, u_{\max }\right), 0\right)
$$

Therefore, using the characterization of the optimal control, we have the following optimality system:

$$
\begin{gathered}
\dot{S}^{*}=A-\left(\mu+u^{*}\right) S^{*}-\frac{\beta S^{*} I^{*}}{1+\alpha_{1} S^{*}+\alpha_{2} I^{*}}, \\
\dot{E}^{*}=\frac{\beta S^{*} I^{*}}{1+\alpha_{1} S^{*}+\alpha_{2} I^{*}}+(\sigma+\mu) E^{*},
\end{gathered}
$$

TABLE 1: Values of the parameters.

\begin{tabular}{ll}
\hline Value & Description \\
\hline$S_{0}=80$ & Initial susceptible population \\
$E_{0}=20$ & Initial exposed population \\
$I_{0}=3$ & Initial infected population \\
$R_{0}=20$ & Initial recovered population \\
$\mu=0.4$ & Natural death of the population \\
$\alpha=0.99$ & Death rate due to disease \\
$\alpha_{1}=0.1$ & Parameter that measures the inhibitory effect \\
$\alpha_{2}=0.5$ & Parameter that measures the inhibitory effect \\
$\beta=0.6$ & Transmission rate \\
$\gamma=0.9$ & Recovery rate \\
$\sigma=0.5$ & The rate at which exposed individuals become \\
$A=100$ & infectious \\
$A_{1}=100$ & Recruitment rate \\
$A_{2}=100$ & Weight parameter \\
$A_{3}=100$ & Weight parameter \\
$\tau=10^{7}$ & Weight parameter \\
\hline
\end{tabular}

$$
\begin{gathered}
\dot{I}^{*}=\sigma E^{*}-(\mu+\alpha+\gamma) I^{*}, \\
\dot{R}^{*}=\gamma I^{*}-\mu R^{*}+u^{*} S^{*}, \\
\dot{\lambda}_{1}=-A_{1}+\lambda_{1}\left(\mu+u^{*}+\frac{\beta I^{*}\left(1+\alpha_{2} I^{*}\right)}{\left(1+\alpha_{1} S^{*}+\alpha_{2} I\right)^{2}}\right) \\
-\lambda_{2}\left(\frac{\beta I^{*}\left(1+\alpha_{2} I^{*}\right)}{\left(1+\alpha_{1} S^{*}+\alpha_{2} I^{*}\right)^{2}}\right)-\lambda_{3} u^{*}, \\
\dot{\lambda}_{2}=-A_{2}+\lambda_{2}(\sigma+\mu)-\lambda_{3}(t) \gamma \\
\dot{\lambda}_{3}=-A_{3}+\lambda_{1}\left(\frac{\beta S^{*}\left(1+\alpha_{1} S^{*}\right)}{\left(1+\alpha_{1} S^{*}+\alpha_{2} I^{*}\right)^{2}}\right) \\
-\lambda_{2}\left(\frac{\beta S^{*}\left(1+\alpha_{1} S^{*}\right)}{\left(1+\alpha_{1} S^{*}+\alpha_{2} I^{*}\right)^{2}}\right) \\
+\lambda_{3}(t)(\mu+\alpha+\gamma)-\lambda_{4} \gamma \\
\dot{\lambda}_{4}=\lambda_{4} \mu
\end{gathered}
$$

with $\lambda_{1}\left(t_{\text {end }}\right)=0, \lambda_{2}\left(t_{\text {end }}\right)=0, \lambda_{3}\left(t_{\text {end }}\right)=0, \lambda_{4}\left(t_{\text {end }}\right)=0$, $S(0)=S_{0}, E(0)=E_{0}, I(0)=I_{0}$, and $R(0)=R_{0}$.

\section{Numerical Simulations}

In this section, we solve numerically the optimality system (16) using the Gauss-Seidel-like implicit finite-difference method developed by Gumel et al. [7], and we use in this simulation the parameter values given in Table 1 .

Figure 1 shows that a significant difference in the number of susceptible, exposed, infected, and recovered individuals 


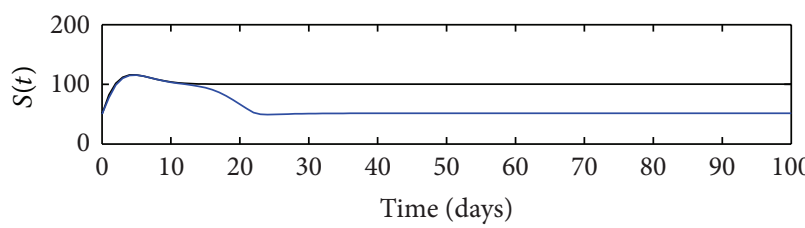

- $S$ without control

- $S$ with control

(a)

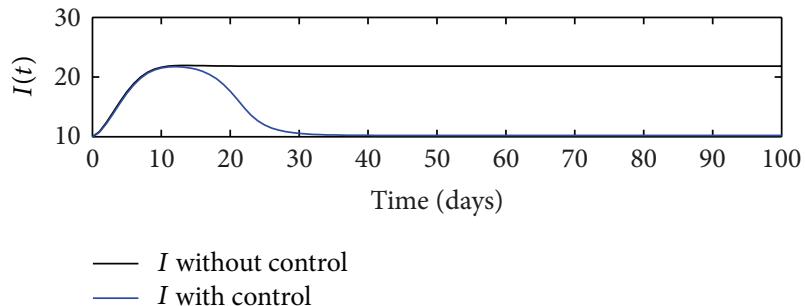

(c)

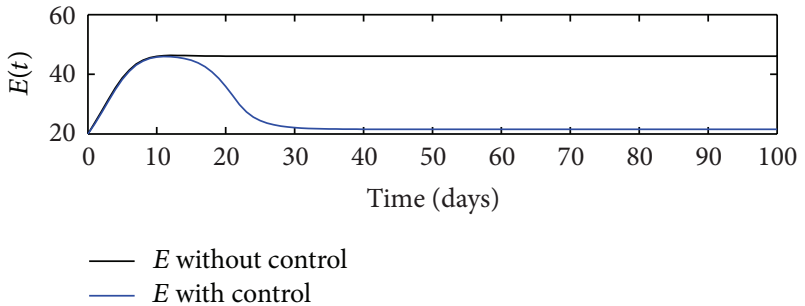

(b)

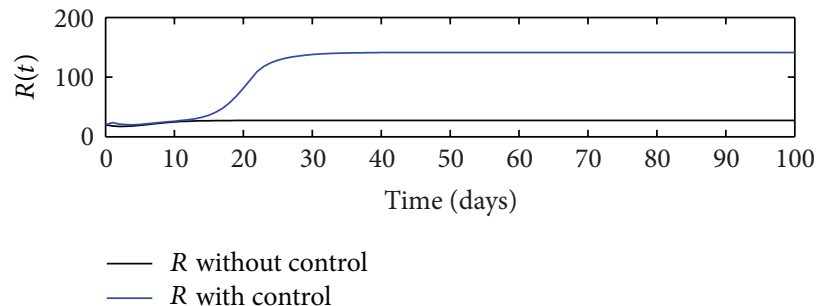

(d)

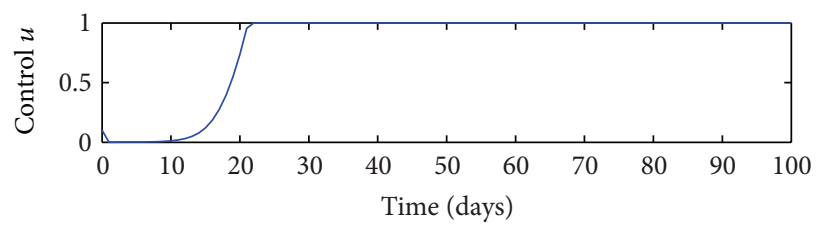

(e)

FIgURE 1: Evolution of different classes of individuals with or without control.

with and without control from the twenty days of vaccination, after that it begins to go to the stable state.

\section{Conclusions and Future Research}

We have presented in this paper the SEIR model with latent period and a modified saturated incidence rate. Our aim is to outline the steps in setting up an optimal control problem, so we presented an efficient numerical method based on optimal control to identify the best vaccination strategy of SEIR model. Our numerical results show that the optimal vaccination strategies for the diseases have a latent period to reduce the number of susceptible, exposed, and infected individuals and increase the number of recovered after twenty days of vaccination.

In future research, we determine the optimal control strategies for the delayed SIR model and compare it with that presented in this work. It is an important subject to study these two types of modeling the incubation period.

\section{References}

[1] H. W. Hethcote, H. W. Stech, and P. van den Driessche, "Periodicity and stability in epidemic models: a survey," in Differential Equations and Applications in Ecology, Epidemics, and Population Problems, S. N. Busenberg and K. L. Cooke, Eds., p. 6582, Academic Press, New York, NY, USA, 1981.
[2] A. Kaddar, A. Abta, and H. T. Alaoui, "A comparison of delayed SIR and SEIR epidemic models," Nonlinear Analysis: Modelling and Control, vol. 16, no. 2, pp. 181-190, 2011.

[3] A. Abta, A. Kaddar, and H. T. Alaoui, "Global stability for delay SIR and SEIR epidemic models with saturated incidence rates," Electronic Journal of Differential Equations, vol. 2012, no. 23, pp. 1-13, 2012.

[4] D. L. Lukes, Differential Equations: Classical to Controlled, vol. 162 of Mathematics in Science and Engineering, Academic Press, New York, NY, USA, 1982.

[5] W. H. Fleming and R. W. Rishel, Deterministic and Stochastic Optimal Control, Springer, New York, NY, USA, 1975.

[6] I. K. Morton and L. S. Nancy, Dynamics Optimization the Calculus of Variations and Optimal Control in Economics and Management, Elsevier Science, Amsterdam, The Netherlands, 2000.

[7] A. B. Gumel, P. N. Shivakumar, and B. M. Sahai, "A mathematical model for the dynamics of HIV-1 during the typical course of infection," in Proceedings of the $3 r d$ World Congress of Nonlinear Analysts, vol. 47, pp. 2073-2083, 2001. 


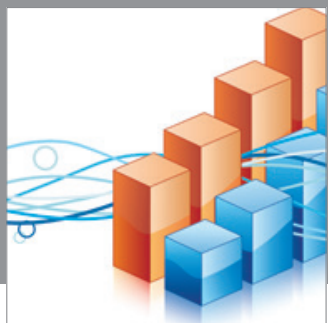

Advances in

Operations Research

mansans

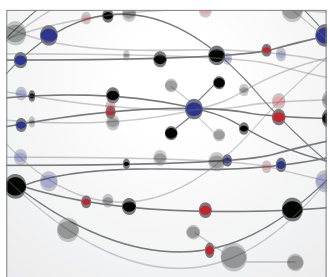

The Scientific World Journal
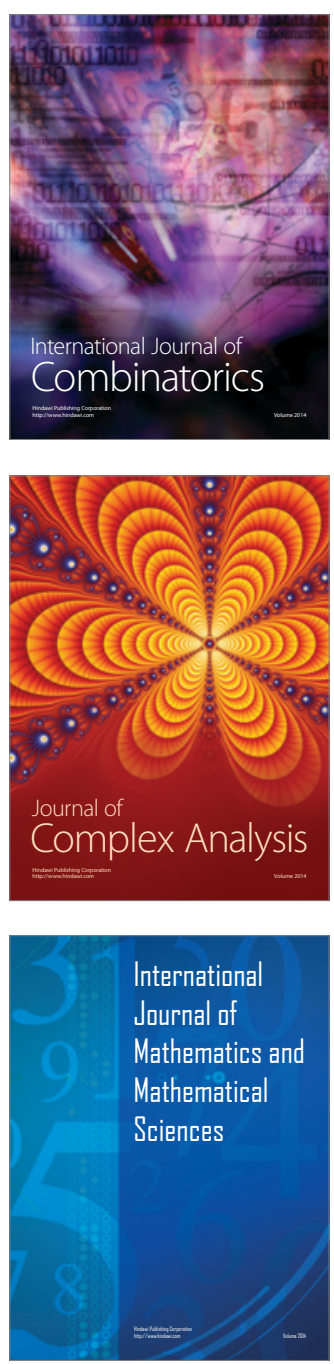
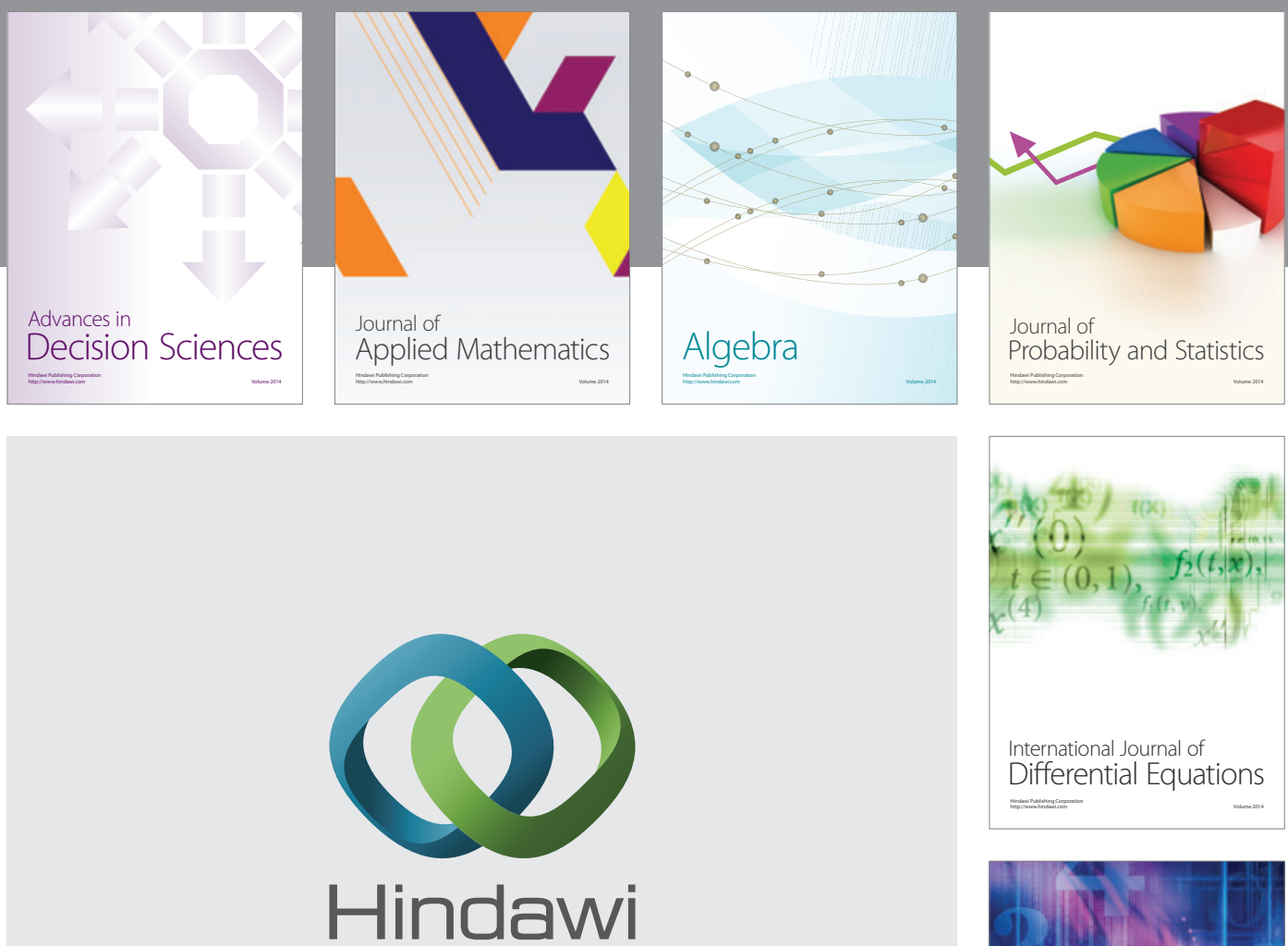

Submit your manuscripts at http://www.hindawi.com
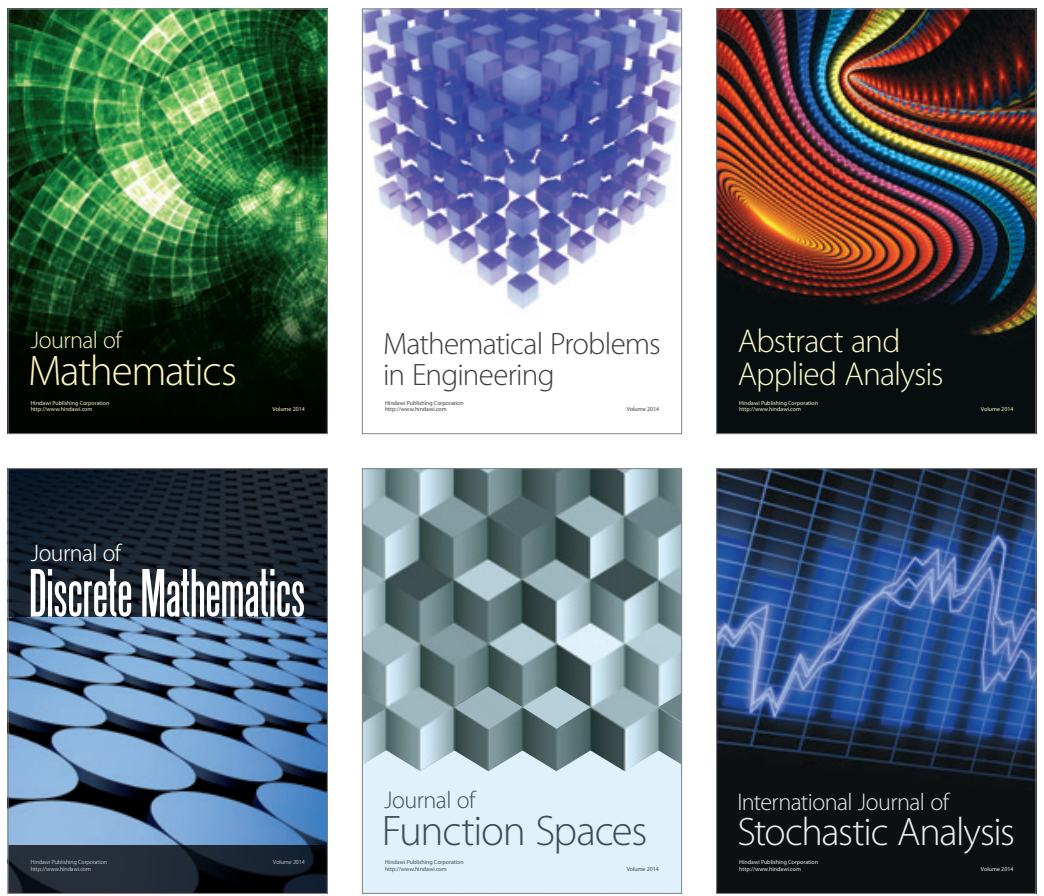

Journal of

Function Spaces

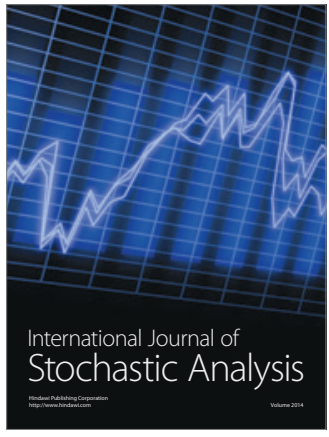

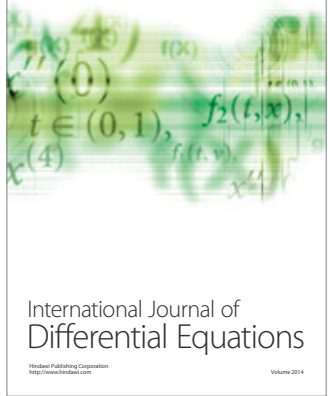
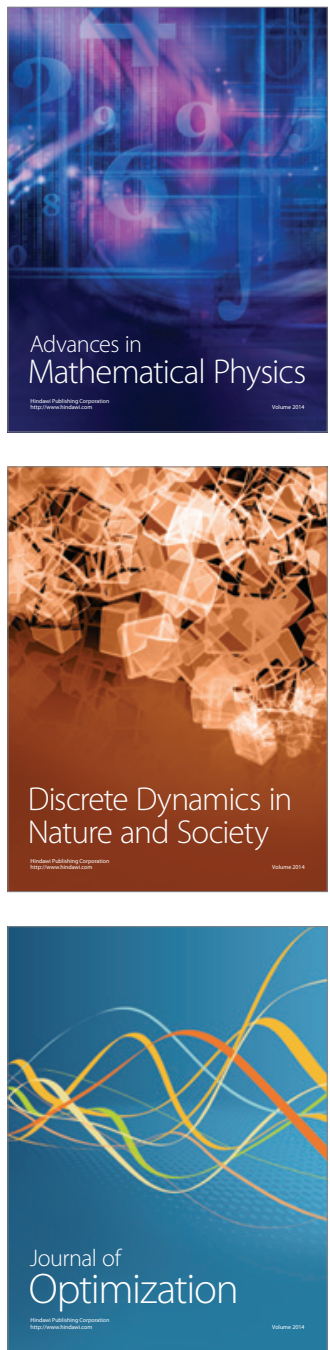